\title{
Impact of stroke unit in a public hospital on length of hospitalization and rate of early mortality of ischemic stroke patients
}

\author{
Impacto da unidade de AVC em hospital público sobre a hospitalização e mortalidade \\ no acidente vascular cerebral isquêmico
}

Maria Sheila G. Rocha, Ana Cláudia F. Almeida, Osório Abath Neto, Marianna P. R. Porto, Sonia Maria D. Brucki

\begin{abstract}
We ascertained whether a public health stroke unit reduces the length of hospitalization, the rate of inpatient fatality, and the mortality rate 30 days after the stroke. Methods: We compared a cohort of stroke patients managed on a general neurology/medical ward with a similar cohort of stroke patients managed in a str oke unit. The in-patient fatality rates and 30-day mortality rates were analyzed. Results: 729 patients were managed in the general ward and 344 were treated at a comprehensive stroke unit. The in-patient fatality rates were $14.7 \%$ for the general ward group and 6.9\% for the stroke unit group ( $p<0.001)$. The overall mortality rate 30 days after stroke was $20.9 \%$ for general ward patients and $14.2 \%$ for stroke unit patients $(p=0.005)$. Conclusions: We observed reduced in-patient fatalities and 30 -day mortality rates in patients managed in the stroke unit. There was no impact on the length of hospitalization.
\end{abstract}

Keywords: mortality, stroke, stroke unit, hospitalization length.

RESUMO

Avaliar o impacto da unidade de AVC (acidente vascular cerebral) no sistema público de saúde sobre o tempo de internação, mortalidade hospitalar e mortalidade após 30 dias do AVC agudo. Métodos: Comparamos uma coorte de pacientes com AVC agudo tratados em enfermaria neurológica ou geral (EG) com uma coorte similar de pacientes com AVC tratados em uma unidade de AVC (UAVC), em um mesmo hospital público. Resultados: 729 pacientes foram conduzidos na EG e 344 foram tratados em uma UAVC. A mortalidade inicial foi de 14,7\% na EG e $6,9 \%$ na UAVC ( $p<0,001)$. A mortalidade geral em 30 dias após o AVC foi de 20.9\% nos pacientes tratados na EG e 14,2\% naqueles tratados na UAVC ( $p=0,005)$. Conclusão: Observamos significante redução da mortalidade inicial e da mortalidade após 30 dias do AVC nos pacientes tratados na UAVC. Não houve impacto sobre o tempo de internação.

Palavras-chave: mortalidade, acidente vascular encefálico, unidade de AVC, tempo de hospitalização.

Stroke unit care is one of the most powerful interventions available to help stroke patients. Several clinical trials have demonstrated the superiority of comprehensive stroke unit care over conventional treatment provided in general hospital wards ${ }^{1-5}$. The effectiveness of stroke units in reducing mortality, institutionalization, and overall dependence has been confirmed in meta-analyses of randomized controlled trials comparing stroke unit care with general ward care $^{2}$. The latest Cochrane review concluded that stroke patients who receive organized in-patient care in a stroke unit are more likely to stay alive, independent, and living at home 1 year after the stroke ${ }^{6}$. Recently, a multicenter observational and prospective study in Spain showed a better outcome at 6 months when patients were admitted to a stroke unit. In addition, patients who received stroke unit care have better functioning and lower rates of recurrent strokes than those treated in a conventional ward ${ }^{5}$.

Although these are important findings, there are still limited data available to assess the impact of stroke units in routine clinical practice outside randomized clinical trials. Additionally, the impact of stroke units on the length of hospitalization is not well characterized. Considering that the

Stroke Team, Department of Neurology, Hospital Santa Marcelina, São Paulo SP, Brazil.

Correspondence: Maria Sheila Guimarães Rocha; Rua Leandro Dupret 364 / Vila Clementino; 04025-011 São Paulo SP - Brazil; E-mail: msrocha@uol.com.br Conflict of interest: There is no conflicts of interest to declare.

Received 22 February 2013; Received in final form 04 July 2013; Accepted 12 July 2013. 
length of hospitalization is an important determinant of inpatient hospital costs, these data are important for policy makers and crucial for public health care systems, especially in underdeveloped countries. A study of a Canadian stroke unit suggested that a 2-day reduction in the length of hospitalization per stroke unit patient translates to savings of more than \$2.1 million per 1000 patients treated?

Despite consensus statements from the Brain Attack Coalition $^{8,9}$ and various national stroke societies in Europe ${ }^{10}$, the United States ${ }^{9,11}$, Canada ${ }^{12}$, Chile ${ }^{13}$, and Brazil ${ }^{14}$ recommending that stroke unit care be widely implemented, organized stroke unit care is not a worldwide reality. Stroke units require significant capital, which may be prohibitively expensive for underdeveloped countries. On the other hand, health authorities should carefully analyze stroke incidence, morbidity, mortality, and the annual costs associated with premature death, long-term disability, restricted social functioning, cost of care, lost productivity, and informal caregiver time. The known benefits of a stroke unit should be seriously taken into account. Unfortunately, stroke units are quite far from being a public health reality in Brazil and in many other South American countries.

Prior to April 2010 at the Santa Marcelina Hospital, a public health system-based university hospital located on the periphery of São Paulo, stroke patients were cared for by both the general neurology service and in the general medical wards. In 2010, a multidisciplinary stroke unit was opened at Santa Marcelina Hospital, where there was a high mortality rate, the highest incidence of stroke in São Paulo, and a lowincome patient population. The hospital serves as a tertiary medical center for a referral population of 4 million people. The stroke unit is a comprehensive, dedicated, high-observation 14-bed unit shared with the Neurology Department. The multidisciplinary staff consists of a nurse practitioner, nurse, neurologist, neurosurgeon, social worker, neuropsychologist, nutritionist, and physical and speech therapists. The stroke team is a dedicated group that is specifically assigned to the stroke unit ward. Stroke thrombolysis was routinely available during the entire study period. The stroke team followed a written multidisciplinary clinical protocol and rehabilitation interventions began immediately after the stroke. The majority of stroke patients were directly admitted to the unit from the emergency department, and all patients with subarachnoid hemorrhage were cared for by the neurosurgery team.

\section{PURPOSE}

We compared the length of hospitalization, in-patient fatalities, and 7-day and 30-day mortality rates between ischemic stroke patients managed in the stroke unit from April 2010 to June 2011 and those managed on the neurology or general wards from December 2008 to March 2010 at the
Santa Marcelina Hospital to assess whether a comprehensive stroke unit had the potential to improve stroke recovery in a public health care system stroke unit.

\section{METHODS}

\section{Study design}

A retrospective study of two cohorts at the Santa Marcelina Hospital, São Paulo, Brazil, was conducted. Data from all patients from our institution who experienced a stroke were collected in a database after December 2008. The main inclusion criteria were acute ischemic stroke (AIS) or transient ischemic attack (TIA) according to the World Health Organization's definition ${ }^{15}$ and admission to the hospital within the first 24 hours after the onset of symptoms. We compared a cohort of stroke patients managed on the general neurology/medical wards in the hospital from December 2008 to March 2010 prior to the initiation of the stroke unit with a similar cohort of stroke patients managed in a stroke unit between April 2010 and June 2011. Once the stroke unit had opened, all stroke patients fulfilling thrombolysis criteria were preferentially admitted to the stroke unit. Treatment of all stroke patients followed a clinical algorithm that included risk evaluation by the nursing staff during admitting, and medical and neurological evaluation and cranial tomography in the emergency room. All stroke patients were under the same clinical and neurological protocol, which included palliative care for those with severe stroke and decompressive craniectomy when indicated by local neurosurgery team. Not all stroke patients were admitted to the stroke unit after April 2010, partly due to the prevalence of strokes in this region and the limited number of available beds in our stroke unit. The decision on whether a patient went to the stroke unit or to the general neurology/medical ward was based solely on stroke unit availability.

Data analyzed included demographic characteristics (e.g. age and gender), risks factors for stroke and comorbid conditions, length of hospitalization, frequency of inpatient mortality, 7- and 30-day mortality rates. Our general hypothesis was that patients with TIA or AIS experience reduced inpatient fatality and 30-day mortality rates, and experienced a shorter length of hospitalization after stroke, if they had received stroke unit treatment rather than general neurology/ medical treatment. The ethics committee of Santa Marcelina Hospital examined and approved the protocol used for this study.

\section{Statistical analysis}

Data management and statistical analyses were performed using the GraphPad Prism Statistical package (Version 5.0 - GraphPad Software-2010). Data are shown using standard descriptive statistics. Continuous variables were 
compared using a Student's $t$-test for normally distributed variables, and categorical variables were compared using a chi-squared $\left(\chi^{2}\right)$ test. Because the distribution of the length of hospitalization was right-skewed in both cohorts, the length of stay was binned into two groups around the median hospitalization length (8 days). The primary outcomes were total length of hospitalization, length of hospitalization $\geq 8$ days or $<8$ days, inpatient fatality rates, 7-day and 30-day mortality rates. A multinomial logistic regression was performed to evaluate the association between stroke care group and mortality in acute ischemic stroke patients with adjustment for age, sex, National Institute Health Stroke Scale (NIHSS) score, and use of thrombolytic therapy; $\mathrm{p}<0.05$ was considered statistically significant.

\section{RESULTS}

Between December 2008 and June 2011, there were 1073 patients with ischemic stroke at our institution. There were 729 patients who were managed on the general neurology/ medical ward and 344 patients who were treated in the comprehensive stroke unit. There were no significant differences in gender distribution or average age of the two cohorts (Table 1). Arterial hypertension was the most frequent risk factor for stroke in both groups. Diabetes mellitus, alcoholism, smoking, and hyperlipidemia were significantly more frequent in stroke unit patients (Table 1). Stroke unit patients were also more likely to have cardiac disease (41.6\% for stroke unit patients compared to $27.3 \%$ for general neurology/medical ward patients: $\chi^{2}=2.85, \mathrm{p}=0.044$ ). In both groups approximately one third of the stroke patients had previously experienced a stroke. Overall, the mean number of risk factors for stroke was significantly higher for the stroke unit group. There was a significantly higher prevalence of patients with transient ischemic attack (TIA) on the general neurology/ medical ward $(14.5 \%)$ than in the stroke unit $(6.2 \%)\left(\chi^{2}=21.96\right.$, $\mathrm{p}<0.001)$.

The mean initial NIHSS score was 8.7 (range 0-47, median 7) for general/neurology ward patients compared to 8.1 (range 0-26, median 6) for the stroke unit patients. This difference was not statistically significant $(\mathrm{p}=0.173)$. We analyzed the proportion of patients in each group, dividing them into three subgroups according to the NIHSS: 1) those with an initial NHISS $\leq 8$ points; 2) those with an initial NIHSS between 9 and 15 points; and 3) those with an initial NIHSS

Table 1. Demographic, clinical, and outcome data of stroke patients.

\begin{tabular}{|c|c|c|c|}
\hline & General Neurology and medical wards & Stroke unit & $p$-value \\
\hline Number of patients & 729 & 344 & \\
\hline Mean age in years (SD) & $65.5(14.7)$ & $67.1(13.1)$ & $0.105^{*}$ \\
\hline Female (\%) & 49.3 & 50.3 & 0.434 \\
\hline NIHSS (Mean) & 8.7 & 8.1 & $0.173^{*}$ \\
\hline 1-8 points (\%) & 57.8 & 66.3 & 0.077 \\
\hline $9-15$ points (\%) & 24.9 & 17.7 & 0.078 \\
\hline$>15$ points (\%) & 17.3 & 15.9 & 0.433 \\
\hline Glasgow Coma Scale (mean) & 12.8 & 13.1 & $0.593 *$ \\
\hline$\leq 8$ points $(\%)$ & 9.8 & 13.9 & 0.296 \\
\hline Thrombolysis (\%) & 5.8 & 12.8 & $<0.001$ \\
\hline $\mathrm{TIA}(\%)$ & 14.5 & 6.2 & $<0.001$ \\
\hline \multicolumn{4}{|l|}{ Risk factors (\%) } \\
\hline Arterial hypertension & 76.7 & 81.5 & 0.059 \\
\hline Diabetes mellitus & 31.7 & 42.8 & $<0.001$ \\
\hline Current smoker & 17.1 & 36.6 & $<0.001$ \\
\hline Alcoholism & 10.2 & 16.5 & 0.013 \\
\hline Hyperlipidemia & 17.1 & 22.6 & 0.031 \\
\hline Any cardiac disease & 27.3 & 45.1 & 0.044 \\
\hline Prior stroke & 31.9 & 33.3 & 0.340 \\
\hline Number of risk factors for stroke & 2.3 & 2.7 & $<0.001$ \\
\hline Length of hospitalization (days) & 10.8 & 10.2 & $0.391 *$ \\
\hline Length of hospitalization <8 days (\%) & 47.2 & 47.3 & 0.498 \\
\hline Case fatality rate $(\%)$ & 14.7 & 6.9 & $<0.001$ \\
\hline Case fatality rate (\%) - Thrombolysis cases excluded & 17.2 & 9.0 & $<0.001$ \\
\hline 7-day mortality (\%) & 13.1 & 3.5 & $<0.001$ \\
\hline 30-day mortality (\%) & 20.9 & 14.2 & 0.005 \\
\hline Lost to follow-up after discharge (\%) & 6.5 & 2.6 & 0.002 \\
\hline
\end{tabular}

NIHSS: National Institute Health Stroke Scale; TIA: transient ischemic attack.

*Student's t-test. 
above 15 points. The proportion of patients in each NIHSS subgroup was similar for both groups.

The mean initial Glasgow Coma Scale (GCS) score for general/neurology ward patients and stroke unit patients was 12.8 and 13.1, respectively. This difference was not statistically significant (Student's $t$-test, $\mathrm{p}=0.593$ ). Similarly, the proportion of patients with a GCS score below 8 points in each group was not significantly different ( $9.8 \%$ for the general/neurology wards compared to $13.1 \%$ for the stroke unit: Yates corrected $\left.\chi^{2}=0.285, \mathrm{p}=0.296\right)$.

Stroke thrombolysis was available to both cohorts of patients, but there was a statistically significant difference between the percentages of patients who received tPA (tissue plasminogen activator) treatment in each group. The percentage of patients in the stroke unit that received tPA treatment was $12.8 \%$ compared with $5.8 \%$ for patients in the general/neurology wards ( $\mathrm{p}<0.001, \chi^{2}$ test). Nevertheless, stroke thrombolysis did not impact on mortality rates in either group considering the multivariable logistic regression analysis performed $(\mathrm{p}=0.355$, coefficient $=0.3209 ; 95 \% \mathrm{CI}-0.3589$ to 1.0008$)$.

The average length of hospitalization for patients in the stroke unit was 10.2 days (range 2-69 days, median 8) compared to 10.8 days (range 3-179 days, median 8) for patients managed on a general/neurology ward. The proportion of stroke unit patients with a length of hospitalization above 8 days was $52.7 \%$, whereas it was $52.8 \%$ for the general ward group. There were no significant differences between the groups in the length of hospitalization. In-patient fatality rate for stroke patients on the general neurology/medical ward was $14.7 \%$ compared to $6.9 \%$ for patients in the stroke unit (absolute difference 7.8\%; $\mathrm{p}<0.001, \chi^{2}$ test).

Stroke unit care significantly reduced inpatient fatalities (relative risk $0.54,95 \% \mathrm{CI} 0.37-0.78$ ). The 7-day mortality rate for stroke patients in the general ward was $13.1 \%$ compared to $3.5 \%$ for patients in the stroke unit. The rates were significantly different $\left(\mathrm{p}<0.001, \chi^{2}\right.$ test) with an absolute risk difference of 9.5\% (CI 6.5-12.5).

The 30-day mortality rate for stroke patients in the general ward was $20.9 \%$ compared to $14.2 \%$ for patients in the stroke unit. The rates were significantly different $\left(p=0.005, \chi^{2}\right.$ test) with an absolute risk difference of $5.7 \%$ and a relative risk difference of $68 \%$ (relative risk $0.67,95 \%$ CI $0.54-0.93$ ).

Due to the significant difference between the percentages of patients who received tPA treatment in the groups, we excluded patients from both groups who received tPA from our analyses. The inpatient mortality rate for stroke unit patients was $9 \%$, whereas it was $17.2 \%$ for patients in the general neurology/medical wards. This represents an absolute difference of $8.2 \%$ ( $\mathrm{p}=0.001, \chi^{2}$ test $)$ and a relative risk difference of $47.7 \%$ (relative risk $0.477,95 \%$ CI $0.47-0.78$ ).

A multinomial logistic regression analysis, with adjustment for age, sex, NIHSS score, and thrombolytic therapy, was performed after excluding all TIA patients because they may have affected mean NIHSS scores at onset in the ischemic stroke patients treated in the stroke unit and general wards in addition to the possible inclusion of TIA mimics. A total of 947 patients (624 patients in general wards and 323 patients in stroke unit) were analyzed. The logistic regression analysis disclosed a significant impact of stroke unit care on mortality: conditioning on all the above possible confounders, the predicted probability of dying after an acute stroke event is $25.9 \%$ lower in those treated in the stroke unit than those treated in general ward (coefficient $=-1.047$, standard error $=0.311$; $\mathrm{z}$ score $=-3.36$; $95 \%$ CI -1.658 to -0.437 ; $\mathrm{p}<0.001$ ).

We were unable to perform follow-up examinations on $6.5 \%$ of stroke patients from the general/neurology ward group after discharge compared to $2.6 \%$ of stroke patients from the stroke unit.

\section{DISCUSSION}

Stroke remains the leading cause of mortality in Brazil. Considering the frequency of death resulting from strokes, strokes should be considered a critical public health problem. However, medical care resources for cerebrovascular disease prevention, treatment, and rehabilitation are unfortunately scarce. The present stroke mortality rates in Brazil are unacceptable considering the success of other Brazilian medical programs devoted to immunizations, AIDS, organ transplantation, and cardiovascular disease. A goal for public health providers suggested by the Brazilian Stroke Society ${ }^{16}$ is to reduce stroke mortality by increasing preventative measures such as controlling hypertension control and by acutely treating stroke sufferers.

The limited number of cerebrovascular disease publications in Brazil contrasts with the recent increase in Brazilian scientific publications ${ }^{17}$, and even fewer studies exist about Brazilian stroke units. Our study of a Brazilian stroke unit showed that stroke unit care, while not able to impact the length of hospitalization, was associated with reduced inpatient fatalities and lowered 30-day mortality rates. This reduction was observed even though stroke unit patients had a higher number of comorbidities (which confer great hardship upon patients), a smaller number of TIA patients, and a similar mean NIHSS score and GCS score at the time of admission, suggesting that the strokes of the patients from the stroke unit were at least as severe as those of the patients from the general neurology/medical ward. It is also worth noting that in both cohorts the same proportion of patients with severe strokes were present according to mean initial NIHSS scores.

One may also argue that the percentage of patients that received tPA treatment was higher in the stroke unit group, and that might explain why this group had a better average outcome. However, a second analysis excluding patients 
who received tPA treatment showed that the stroke unit group patients still exhibited a significantly improved prognosis. Moreover, the observed reduction in fatalities under both analyses was greater than the $5 \%$ reduction in mortality rates that was seen in other large observational studies from Canada and Italy following stroke unit care ${ }^{4,18}$.

The stroke unit effect on stroke recovery is partly due to the organization of care. This organization improves care, hastens recovery, reduces the need for inpatient rehabilitation, and prevents most clinical complications through effective measures that prevent infections and profound venous thrombosis. From a public health care policy and economic perspective, a shorter length of hospitalization in the stroke unit is promising as the principal cost of care is related to per diem hospitalization costs. However, the development of a stroke unit run by a multidisciplinary team demands a significant economic investment. Nevertheless, a shorter length of hospitalization must not come at the expense of patient outcome.

Another Brazilian study failed to show an impact of stroke unit care on the length of hospitalization and morbidity, although the number of patients analyzed was too small to draw firm conclusions ${ }^{19}$. In the same vein, the Cochrane review did not observe any systematic increase or reduction in the length of hospitalization following stroke unit care ${ }^{6}$. On the other hand, a few studies have shown a positive impact on the length of hospitalization ${ }^{20}$. A study conducted on patients from a stroke unit in Chile also demonstrated a reduction in the length of hospitalization, but there was no impact on the costs associated with treating the strokes. In fact, there was a slight overall increase in hospital costs ${ }^{21}$.

The main objective of a stroke unit is to reduce morbidity and mortality, and economic analyses should be conducted with close attention to the full chain of care and should not focus solely on the length of hospitalization. Although we did not observe a reduction in the length of hospitalization, it is also true that the length of hospitalization did not increase. Additionally, studies that consider the costs associated with the treatment of strokes in South America do consider the impact of acute stroke complications (especially medical complications like pulmonary and urinary infections) on overall $\operatorname{cost}^{22,23}$. Stroke units are supposed to decrease these complications and therefore the ultimate costs associated with strokes. More studies are needed to evaluate the impact of a stroke unit on hospital costs not related to the length of hospitalization. The overall positive impact of the stroke unit on mortality rates and the unquestioned reduction in 30-day mortality rates suggest that we have not made a trade-off in reducing the length of hospitalization only to have patients die in their homes. These reductions were observed despite more number of comorbidities in patients from the stroke unit. Unfortunately, we did not have any classification of disability (Rankin scale score, for example) from patients that have been discharged from general wards to compare with stroke unit patients.

We observed reduced inpatient fatalities, and 7-day and 30-day mortality rates in patients managed in a stroke unit when compared to patients managed in a general neurology/medical ward. There was no significant impact on the length of hospitalization. Our observations support the view that a multidisciplinary stroke unit, which focuses on acute care, reduction of medical complications like pneumonia and deep venous thrombosis, and also immediate neurological rehabilitation, has an effect on reducing early mortality. This effect is large and implies a potential for saving lives, which should be sufficient to encourage governmental administrators and health care providers to endorse a policy of developing stroke units with specialized neurological care for stroke patients in Brazil.

\section{References}

1. Collaborative systematic review of the randomized trials of organised inpatient (stroke unit) care after stroke. Stroke Unit Trialists' Collaboration. BMJ 1997;314:1151-1159.

2. Organised inpatient (stroke unit) care for stroke. Stroke Unit Trialists' Collaboration. Cochrane Database Syst Rev 2000:CD000197.

3. Langhorne P, Lewsey JD, Jhund PS, et al. Estimating the impact of stroke unit care in a whole population: an epidemiological study using routine data. J Neurol Neurosurg Psychiatr 2010;81: 1301-1305.

4. Candelise L, Gattinoni M, Bersano A, et al. Stroke-unit care for acute stroke patients: an observational follow-up study. Lancet 2007; 369:299-305.

5. Alvarez-Sabín J, Ribó M, Masjuan J, Tejada JR, Quintana M, en nombre de los investigadores del estudio PRACTIC. Hospital Care of Stroke Patients: Importance of Expert Neurological Care. Neurologia 2011;26:510-517.

6. Stroke Unit Trialists' Collaboration. Organised inpatient (stroke unit) care for stroke. Cochrane Database Syst Rev 2007:CD000197.
7. Phillips SJ, Eskes GA, Gubitz GJ, Queen Elizabeth II Health Sciences Centre Acute Stroke Team. Description and evaluation of an acute stroke unit. CMAJ 2002;167:655-660.

8. Alberts MJ, Hademenos G, Latchaw RE, Jagoda A, et al. Recommendations for the establishment of primary stroke centers. Brain Attack Coalition. JAMA 2000;283:3102-3109.

9. Alberts MJ, Latchaw RE, Selman WR, et al. Recommendations for comprehensive stroke centers: a consensus statement from the Brain Attack Coalition. Stroke 2005;36:1597-1616.

10. Kaste M, Skyhoj Olsen T, Orgogozo J, Bogousslavsky J, Hacke W. Organization of stroke care: education, stroke units and rehabilitation. European Stroke Initiative (EUSI). Cerebrovasc Dis 2000;10:1-11.

11. Adams HP, del Zoppo G, Alberts MJ, et al. Guidelines for the early management of adults with ischemic stroke: a guideline from the American Heart Association/American Stroke Association Stroke Council, Clinical Cardiology Council, Cardiovascular Radiology and Intervention Council, and the Atherosclerotic Peripheral Vascular Disease and Quality of Care Outcomes in Research Interdisciplinary 
Working Groups: the American Academy of Neurology affirms the value of this guideline as an educational tool for neurologists. Stroke 2007;38:1655-1711.

12. Wilson E, Taylor G, Phillips S, et al. Creating a Canadian stroke system. CMAJ 2001;164:1853-1855.

13. Lavados PM, Hoppe WA. Stroke units in Chile. Rev Med Chil 2005;133:1271-1273.

14. Massaro AM: Stroke in Brazil: a South America perspective. Int $J$ Stroke 2006;1:113-115.

15. Stroke-1989. Recommendations on stroke prevention, diagnosis, and therapy. Report of the WHO Task Force on Stroke and other Cerebrovascular Disorders. Stroke 1989;20:1407-1431.

16. Brazilian Cerebrovascular Disease Society. First Brazilian consensus for the management of the acute phase of cerebral vascular accidents. Arq Neuropsiquiatr 2001;59:972-980.

17. King D. The scientific impact of nations. Nature 2004;430:311-316.
18. Zhu HF, Newcommon NN, Cooper ME, et al. Impact of a stroke unit on length of hospital stay and in-hospital case fatality. Stroke 2009;40:18-23.

19. Cabral NL, Moro C, Silva GR, Scola RH, Werneck LC. Study comparing the stroke unit outcome and conventional ward treatment: a randomized study in Joinville, Brazil. Arq Neuropsiquiatr 2003;61:188-193.

20. Diez-Tejedor E, Fuentes B. Acute care in stroke: do stroke units make the difference? Cerebrovasc Dis 2001;11:31-39.

21. Díaz TV, Illanes DS, Reccius MA, et al. [Evaluation of a stroke unit at a university hospital in Chile]. Rev Med Chile 2006;134:1402-1408.

22. Christensen MC, Valiente R, Sampaio Silva G, et al. Acute treatment costs of stroke in Brazil. Neuroepidemiology 2009;32:142-149.

23. Christensen MC, Previgliano I, Capparelli FJ, Lerman D, Lee WC, Wainsztein NA. Acute treatment costs of intracerebral hemorrhage and ischemic stroke in Argentina. Acta Neurol Scand 2009;119:246-253. 